\title{
Promoção da amamentação em localidade urbana da região sul do Brasil: estudo de intervenção randomizado*
}

\author{
A randomized intervention trial to increase breast-feeding prevalence in \\ southern Brazil
}

Fernando C. Barros ${ }^{\star *}$, Ricardo Halpern ${ }^{\star \star *}$, Cesar G. Victora**, Ana Maria B. Teixeira ${ }^{\star \star}$, Jorge U. Béria**

\begin{abstract}
BARROS, F.C. et al. Promoção da amamentação em localidade urbana da região sul do Brasil: estudo de intervenção randomizado. Rev. Saúde Publica, 28: 277-83, 1994. Foi realizado em Pelotas, RS, Brasil, estudo de intervenção randomizado, para a promoção do aleitamento materno. Grupo de 450 mães e lactentes foram visitados em casa aos 5,10 e 20 dias após o nascimento, e comparados com um grupo-controle do mesmo número. Noventa e dois por cento das familias no grupo de intervenção receberam as três visitas planejadas. A avaliação do padrão de aleitamento materno $e$ causas de desmame aconteceu seis meses depois do nascimento para ambos os grupos. Noventa e quatro por cento do grupo de intervenção e noventa e dois por cento do grupo-controle foram encontrados na visita de avaliação. A intervenção aumentou a duração do aleitamento (mediana de 120 dias no grupo de intervenção, contra 105 dias no grupo-controle; $p=0,03$ ) e retardou a introdução do leite artificial (mediana de idade de 90 dias no grupo de intervenção e 60 dias no grupo-controle; $\mathrm{p}=0,01$ ). As causas de desmame foram classificadas como subjacentes, intermediárias, e imediatas. A causa subjacente mais comum foi "o bebê chora muito", sugerindo que as mães devem ser instruídas a respeito dos padrões normais de comportamento do lactente nas primeiras semanas de vida, em particular da necessidade que a criança tem de chorar e o fato de que isto, não necessariamente, significa fome.
\end{abstract}

Descritores: Aleitamento materno. Promoção da saúde, métodos. Estudos de intervenção.

\section{Introdução}

O aleitamento materno tem forte papel protetor contra a morbidade e mortalidade infantil devido a sua influência em um grande número de doenças principalmente as infecciosas ${ }^{10,19}$. Portanto, o aleitamento materno é essencial para a sobrevivência de crianças pobres em países pouco desenvolvidos, que estão em risco constante de adoecerem devido a complexa associação de má nutrição, falta de saneamento, infecções frequientes e falta de alimentação nutritiva.

Em Pelotas (Estado do Rio Grande do Sul, Brasil, pop. 300.000 ) a situação em relação à alimentação e saúde é causa de preocupação.

* Financiado pela Secretaria de Saúde da Prefeitura Municipal de Pelotas.

** Departamento de Medicina Social da Universidade Fe. deral de Pelotas- Pelotas, RS - Brasil

*** Departamento Materno-Infantil da Universidade Federal de Pelotas - Pelotas, RS - Brasil

Separatas/Reprints: F.C.Barros - Caixa Postal 464 - $96001-970$ - Pelotas, RS - Brasil. FAX (0532) 71.2645 .
Pesquisa realizada em 1982-84 mostrou um coeficiente de mortalidade infantil (CMI) de 40 por mil nascidos vivos, o que representava metade do índice para o país como um todo -70 por mil. Entretanto, para famílias de baixa ren$\mathrm{da}$, que viviam em favelas peri-urbanas, a CMI foi maior que 80 por mil ${ }^{20}$. As mortes infantis nesse grupo da população ocorreram muito precocemente, $90 \%$ delas nos primeiros 6 meses de vida. Além disso, $30 \%$ da mortalidade foi causada por doenças infecciosas preveníveis ${ }^{5}$.

A duração do aleitamento materno nessa população é muito baixa (mediana de 3,3 meses), sendo mais baixa entre famílias de baixa renda $(3,1 \mathrm{me}$ ses) do que entre as famílias mais abastadas $(4,4$ meses) $)^{4}$. Além disso, o aleitamento materno exclusivo é bastante raro devido à introdução precoce de chás nos primeiros dias de vida do bebê, por quase a totalidade das mães. Esta situação também é preocupante porque a alimentação do lactente com leite materno e chás e/ou água pode aumentar o risco de doença diarréica ${ }^{9,21}$.

Com o objetivo de melhorar os padrões de aleitamento materno entre lactentes de baixa renda foi realizado estudo de intervenção randomizado, através de visitas domiciliares, promovidas após o 
nascimento, por profissionais da área da saứde previamente treinados. O objetivo do presente artigo é mostrar o impacto desta intervenção nos padrões de amamentação e na introdução de leites artificiais; e descrever os problemas mais importantes relatados pelas mães para a continuidade da amamentaçăo, o tipo de auxílio oferecido pelas visitadoras e as causas de desmame.

\section{População e Método}

Durante 15 meses (janeiro 1988 - março 1989) todas as mães que tiveram filho nos hospitais de Pelotas foram convidadas para participar do estudo. Entre elas foram selecionadas aquelas que satisfizessem os seguintés critérios de inclusão: a) renda mensal da família menor que dois salários mínimos; b) permanência no hospital menor que 5 dias; c) desejo de amamentar seu bebê; e d) morar em área urbana. Novecentas mães concordaram em participar e após darem o consentimento oral responderam um questionário que continha informações sobre saúde, características socioeconômicas e demográficas. Nos casos em que a mãe tinha experiência prévia de amamentação, um completo relato desta experiência era coletada.

Os sujeitos (mães e bebês) dos grupos de controle e intervenção foram aleatoriamente sorteados por um membro da equipe que não teria nenhum contato posterior com as famílias. A intervenção consistiu de três visitas domiciliares realizadas aos 5,10 e 20 dias após o nascimento. As entrevistadoras foram assistentes sociais ou nutricionistas que haviam tido experiência pessoal bem sucedida com amamentação. Foi realizado treinamento sobre a fisiologia da lactação, as dificuldades mais comuns que a mãe encontra para amamentar e suas melhores soluções.

Após cada visita domiciliar um questionário era preenchido pela entrevistadora com informações relacionadas ao aleitamento materno e a melhor maneira de solucionar os problemas relacionados com a amamentação. Durante o processo de intervenção, os entrevistadores foram supervisionados por um pediatra, e quando necessário outros profissionais de diferentes áreas (obstetra, clínico geral) foram consultados para solucionar problemas específicos.

Ambos os grupos de intervenção e controle foram avaliados 6 meses após o nascimento por uma enfermeira que desconhecia os objetivos do estudo. Embora a enfermeira não recebesse nenhuma informação a respeito das visitas de intervenção, foi impossível mantê-la completamente sem conhecimento do assunto já que as famílias comentavam a respeito das visitas anteriores. O objetivo da visita foi coletar informação sobre aleitamento materno, quando o desmame ocorreu, e avaliar suas causas com um conjunto de questões abertas e fechadas. Com esta informação, dois dos autores (F.C.B. e A.M.B.T.) reconstruíram, independentemente, a história de desmame, categorizando as causas como subjacente, intermediária e imediata. Quando não houve concordância entre as análises sobre desmame, os dois autores revisavam em conjunto a informação para chegar a um consenso.

A análise foi baseada na "intenção de tratar (ou visitar)", o que significa que foi considerado que todos os pares mães-bebês do grupo de intervenção receberam três visitas, mesmo que alguns deles recusassem a continuar no estudo ou não pudessem ser localizados. Foi conduzida uma análise de sobrevivência da incidência e duração do aleitamento materno através do EPILOG ${ }^{13}$; e a regressão de Cox foi usada para controlar possíveis variáveis de confusão.

\section{Resultados}

Das 450 mães e bebês sorteados incialmente para o grupo de intervenção, 412 (92\%) receberam três visitas domiciliares. As proporções de perdas em cada visita podem ser vistas na Tabela 1 ,

Tabela 1. Proporção de crianças acompanhadas e perdidas durante a intervenção para promoção do aleitamento materno.

\begin{tabular}{|c|c|c|c|c|}
\hline \multirow[t]{2}{*}{ Atividade } & \multicolumn{2}{|c|}{ Intervenção } & \multicolumn{2}{|c|}{ Controle } \\
\hline & $\begin{array}{l}\text { Acompa- } \\
\text { nhadas }\end{array}$ & - Perdidas & $\begin{array}{c}\text { Acompa- } \\
\text { nhadas }\end{array}$ & Perdidas \\
\hline $\begin{array}{l}\text { Entrevista } \\
\text { hospitalar }\end{array}$ & 450 & - & 450 & - \\
\hline $\begin{array}{l}\text { Visita } \\
\text { domiciliar } 1\end{array}$ & 431 & $19(4,2 \%)^{\star}$ & - & - \\
\hline $\begin{array}{l}\text { Visita } \\
\text { domiciliar } 2\end{array}$ & 425 & $25(5,5 \%)^{* *}$ & $\cdot$ & $\cdot$ \\
\hline $\begin{array}{l}\text { Visita } \\
\text { dimiciliar } 3\end{array}$ & 412 & $38(8,4 \%)^{\star \star \star}$ & - & - \\
\hline Avaliação & 424 & $26(5,7 \%)$ & 414 & $36(8 \%)$ \\
\hline $\begin{array}{l}\text { * } 19 \text { perda } \\
\text { ** perda } \\
4 \text { criança } \\
\text { domiciliai } \\
\text { *** perda } \\
12 \text { crianç } \\
\text { domicilial }\end{array}$ & $\begin{array}{l}\text { s: } 4 \text { recusa } \\
\text { s: } 4 \text { recusa } \\
\text { s haviam } \\
\text { prévia. } \\
\text { s: } 4 \text { recusa } \\
\text { as haviam } \\
\text { prévia. }\end{array}$ & $\begin{array}{l}\text { as e } 15 \text { crian } \\
\text { as , } 17 \text { crians } \\
\text { parado a al } \\
\text { as, } 22 \text { criano } \\
\text { parado a a }\end{array}$ & $\begin{array}{l}\text { não enco } \\
\text { não enco } \\
\text { mentação } \\
\text { não enco } \\
\text { mentação }\end{array}$ & $\begin{array}{l}\text { ontradas. } \\
\text { ontradas e } \\
\text { na visita } \\
\text { ontradas e } \\
\text { o na visita }\end{array}$ \\
\hline
\end{tabular}


Tabela 2. Características da população estudada.

\begin{tabular}{|c|c|c|c|c|}
\hline \multirow{2}{*}{$\begin{array}{l}\text { Característica In } \\
\text { Idadematerna(média) } \\
<20 \text { anos }\end{array}$} & \multicolumn{2}{|c|}{ Intervenção Controle } & \multicolumn{2}{|c|}{ Diferença $(95 \% \mathrm{Cl})$} \\
\hline & $\begin{array}{l}25,1 \\
23,8 \%\end{array}$ & $\begin{array}{l}25,4 \\
20,0 \%\end{array}$ & $\begin{array}{l}-0,31 \\
3,8\end{array}$ & $\begin{array}{l}(-1,19,0,57) \\
(-1,6,9,2)\end{array}$ \\
\hline Mãe solteira & $16,4 \%$ & $14,4 \%$ & 2,0 & $(-2,7,6,7)$ \\
\hline $\begin{array}{l}\text { Fumo durante a ges- } \\
\text { tação }\end{array}$ & $41,3 \%$ & $42,4 \%$ & $-1,1$ & $(-7,5,5,3)$ \\
\hline $\begin{array}{l}\text { Pré-natal (média) não } \\
\text { realizou }\end{array}$ & $\begin{array}{l}7,0 \\
5,3 \%\end{array}$ & $\begin{array}{l}6,9 \% \\
4,0 \%\end{array}$ & $\begin{array}{l}0,2 \\
1,3\end{array}$ & $\begin{array}{l}(-0,2,0,6) \\
(-1,5,4,0)\end{array}$ \\
\hline $\begin{array}{l}\text { Escolaridade do pai } \\
\text { (anos) }\end{array}$ & & & & \\
\hline $\begin{array}{l}\text { média (*) } \\
\text { não frequentou es- } \\
\text { cola }\end{array}$ & $\begin{array}{l}5,2 \\
9,2 \%\end{array}$ & $\begin{array}{c}4,8 \\
10,4 \%\end{array}$ & $\begin{array}{r}0,4 \\
-1,2\end{array}$ & $\begin{array}{l}(0,0,0,8) \\
(-5,2,2,8)\end{array}$ \\
\hline $\begin{array}{l}\text { Escolaridade da mãe } \\
\text { (anos) }\end{array}$ & & & & \\
\hline $\begin{array}{l}\text { média ("*) } \\
\text { não frequentou es- } \\
\text { cola }\end{array}$ & $\begin{array}{l}5,2 \\
4,0 \%\end{array}$ & $\begin{array}{l}4,6 \\
6,0 \%\end{array}$ & $\begin{array}{l}0,6 \\
-2,0\end{array}$ & $\begin{array}{l}(0,3,0,9) \\
(-4,8,08)\end{array}$ \\
\hline $\begin{array}{l}\text { Peso de nascimento } \\
\text { média }(\mathrm{g})\end{array}$ & 3,173 & 3,152 & 21 & $(-44,86)$ \\
\hline $\begin{array}{l}\text { Idade gestacional } \\
\text { média (semanas) }\end{array}$ & 40,0 & 39,7 & 0,3 & $(-0,05,0,65)$ \\
\hline Cesariana & $28,2 \%$ & $22,9 \%$ & 5,3 & $(0,4,11,0)$ \\
\hline $\begin{array}{l}\text { Sem familiares durante } \\
\text { o trabalho de parto }\end{array}$ & $7,1 \%$ & $8,7 \%$ & $-1,6$ & $(-5,1,1,9)$ \\
\hline $\begin{array}{l}\text { Sem famillares du- } \\
\text { rante o nascimento }\end{array}$ & $99,1 \%$ & $99,3 \%$ & $-0,2$ & $(-1,4,1,0)$ \\
\hline $\begin{array}{l}\text { Sem familiares no pri- } \\
\text { meiro dia pos-parto } \\
\text { Alojamento conjunto }\end{array}$ & $\begin{array}{l}25,8 \% \\
93,6 \%\end{array}$ & $\begin{array}{l}25,8 \% \\
93,8 \%\end{array}$ & $\begin{array}{c}0 \\
-0,2\end{array}$ & $\begin{array}{l}(-5,7,5,7) \\
(-3,4,3,0)\end{array}$ \\
\hline $\begin{array}{l}\text { Criança fol colocada } \\
\text { no selo antes da en- } \\
\text { trevista }\end{array}$ & $86,2 \%$ & $89,8 \%$ & $-3,6$ & $(-7,8,0,6)$ \\
\hline $\begin{array}{l}\text { Amamentou anterior- } \\
\text { mente } \\
\text { média (meses) } \\
\text { não amamentou }\end{array}$ & $\begin{array}{l}7,7 \% \\
7,6 \%\end{array}$ & $\begin{array}{l}8,7 \\
8,0 \%\end{array}$ & $\begin{array}{l}-0,95 \\
-0,4\end{array}$ & $\begin{array}{l}(-2,80,0,90) \\
(-4,9,4,1)\end{array}$ \\
\hline $\begin{array}{l}\text { Morte prévia de um } \\
\text { filho }\end{array}$ & $7,6 \%$ & $11,8 \%$ & $-4,2$ & $(-8,1,0,3)$ \\
\hline
\end{tabular}

* $p<0,05$

** $p<0,01$

mostrando que as perdas durante a avaliação foram de $6 \%$ no grupo de intervenção e $8 \%$ no grupo - controle.

As principais características da população estudada são mostradas na Tabela 2.

Mesmo com a distribuição aleatória dos sujeitos, o grupo de intervenção apresentou maior nível de escolaridada dos pais. Por outro lado, alta pro- porção de mulheres do grupo-controle relataram a morte de algum filho.

\section{Impacto da intervenção nos padrões de aleitamento}

A Figura mostra que a intervenção aumentou a prevalência e duração do aleitamento materno. Com um mês de idade $86 \%$ dos bebês do grupo de intervenção foram amamentados, comparados com $77 \%$ no grupo controle. Estas proporções foram, respectivamente, $73 \%$ e $62 \%$ aos dois meses de vida. A mediana da duração do aleitamento materno no grupo de intervenção foi de 120 dias, enquanto que no grupo controle foi de 105 dias.

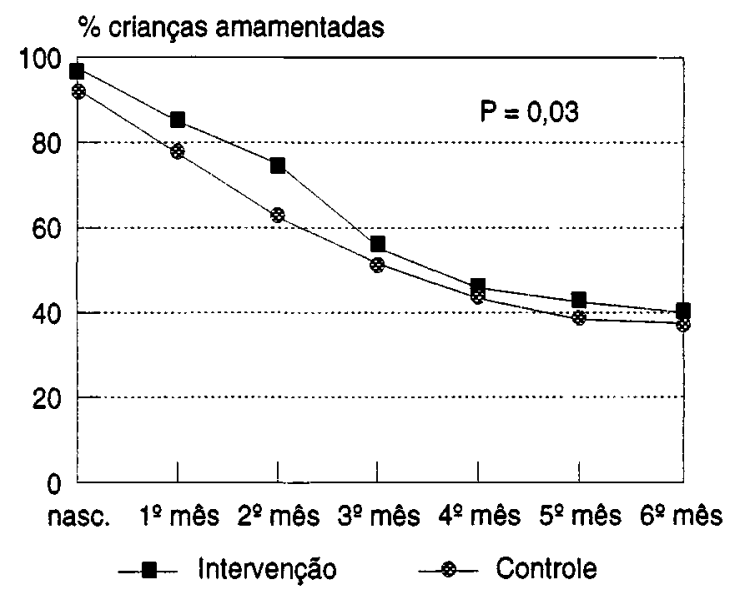

Pelotas, RS, Brasil, 1988-89.

Figura. Proporção de crianças amamentadas nos grupos de intervenção e controle até o $6^{\circ}$ mês de vida.

A intervenção realizada conseguiu retardar a introdução de leite artificial. Com um mês de idade, $36 \%$ das crianças do grupo controle já haviam começado com mamadeira, em comparação com $22 \%$ no grupo de intervenção; e com dois meses as proporções eram $53 \%$ e $40 \%$, respectivamente. A mediana de idade de introdução de mamadeira foi de 90 dias para o grupo de intervenção e 60 dias para o grupo-controle.

A intervenção não teve nenhum impacto no tempo em que foi introduzido água (mediana de idade de 90 dias para ambos os grupos), sucos de frutas (mediana de idade de 112 e 114 dias no grupo de intervenção e controle), papa de frutas e de vegetais (mediana de idade de 120 dias em ambos os grupos). Algumas variáveis distribuídas diferentemente nos grupos de intervenção e controle poderiam confundir a asso- 
ciação entre intervenção e os padrões de aleitamento. As variáveis foram: a) tempo de introdução de chás caseiros; b) educação dos pais; e c) morte prévia de um filho. Mesmo com a introdução um pouco mais tardia de chás no grupo de intervenção do que no grupo controle (mediana de idade de 5 e 3 dias respectivamente, $p<0,01$ ), não foram encontradas diferenças nos padrões de aleitamento entre os bebês que receberam chás antes ou depois de 5 dias de vida. Da mesma maneira, não foi encontrada associação entre a duração do aleitamento materno e história de perda prévia de um filho. Finalmente, a educação dos pais também não mostrou associação com a duração do aleitamento. Quando os efeitos de educação materna e paterna foram controlados, não houve mudanças substanciais nas razões de chance na regressão logística, mesmo depois de incluir educação dos pais no modelo. Portanto essas variáveis não devem ser consideradas como variáveis de confusão na associação de aleitamento materno e intervenção.

Dificuldades encontradas pelas mães durante a amamentação $e$ aconselhamento oferecido pelos visitadores

Os problemas mais freqüentes relatados pelas mães durante as visitas domiciliares foram fissuras mamárias, engurgitamento mamário, mamilo plano, dor no seio, leite insuficiente e leite fraco. A Tabela 3 mostrou que $85 \%$ das mães necessitaram ajuda durante a primeira visita domiciliar, $83 \%$ na segunda e $75 \%$ na terceira. A Tabela também mostra o tipo de aconselhamento oferecido pelos visitadores em cada visita. As

Tabela 3. Orientação oferecida pelos visitadores durante cada visita domiciliar.

\begin{tabular}{lrrr}
\hline Tipo de orientação & $\begin{array}{r}\text { Visita } 1 \\
(\%)\end{array}$ & $\begin{array}{r}\text { Visita 2 } \\
(\%)\end{array}$ & $\begin{array}{r}\text { Visita 3 } \\
(\%)\end{array}$ \\
\hline Amamentar mais seguidamente & 20,5 & 22,0 & 22,7 \\
Compressas mornas no seio & 15,4 & 4,8 & 2,6 \\
Retardar o uso da mamadeira & & 12,1 & 8,1 \\
e/ou chás & 10,8 & 12,1 & 10,4 \\
Melhora da alimentação materna & 8,9 & 6,5 & 10,9 \\
Ensinara colocaro bebê no selo & 8,4 & 6,2 & 2,9 \\
Orientação de higiene & 3,2 & 13,6 & 10,4 \\
Orientação quando o bebê tem & 3,0 & 8,5 & 6,5 \\
cólica & 2,7 & 8,2 & 9,7 \\
Continuar a amamentação & 3,0 & 4,0 & 13,0 \\
Levar o bebê ao médico & 85,8 & 83,3 & 75,0 \\
\hline \% que necessitou ajuda & $(370)$ & $(354)$ & $(309)$ \\
\hline Total & & &
\end{tabular}

mais freqüentes orientações dadas pela visitadora foram: a) encorajar as mães a colocar o filho mais seguidamente no seio. b) usar compressas mornas para aliviar o engurgitamento mamário c) desencorajar a introdução de chás e/ou leite artificial.

\section{Causas de desmame}

A Tabela 4 mostra que a causa subjacente mais importante para o desmame foi o "bebê chora muito" $(38,2 \%)$, seguido por "o leite secou" $(16,0 \%)$. A causa intermediária mais freqüente foi "iniciou com mamadeira" $(26,1 \%)$ e o "leite secou" $(21,0 \%)$. Em relação à causa imediata, "iniciou com mamadeira" foi a causa mais comum $(56,2 \%)$, seguida por "o bebê não quer mais o seio" $(17,1 \%)$. A ordem mais comum dos eventos que levaram ao desmame foi: o bebê chorava muito, as mães (e famílias) atribuíram o choro à fome, concluindo que o leite matermo estava sendo insuficiente ou fraco $e$ in-

Tabela 4. Causas de desmame.

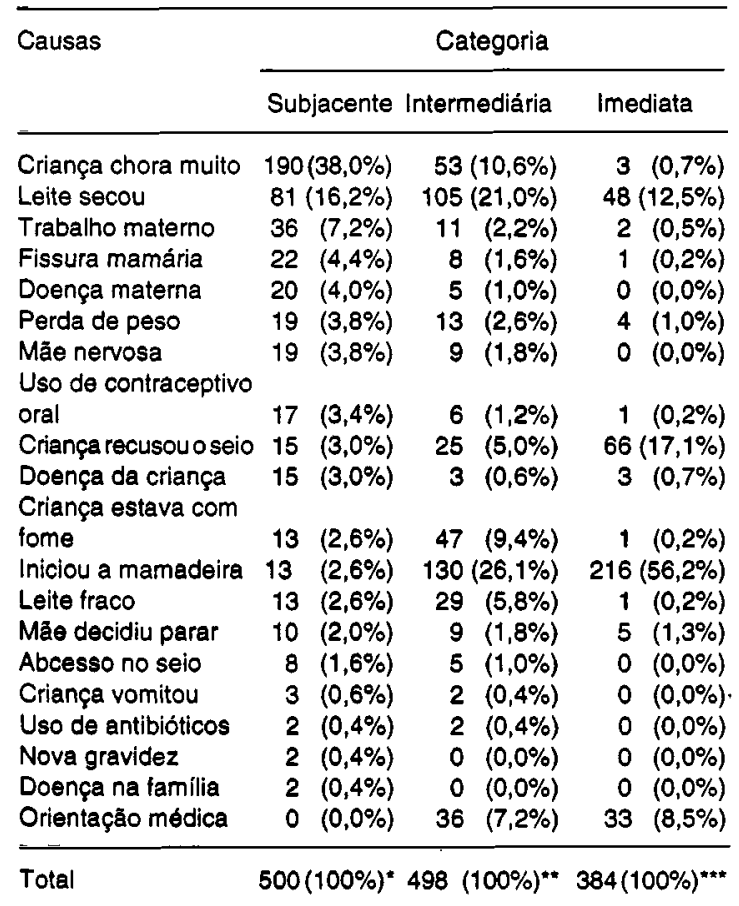

- Para as 400 crianças restantes, 336 estavam sendo amamentadas e em 64 delas a causa do desmame năo ficou definida.

* Para as 402 crianças restantes, 336 ainda estavam sendo amamentadas e em 64 delas a causa do desmame não ficou definida. Em 2 casos não foi relatado causa intermediária.

** Para as 516 crianças restantes, 336 ainda estavam sendo amamentadas e para 64 delas a causa do desmame não ficou definida. Em 116 casos não foi relatado causa imediata. 
iciaram alimentação com leite artificial, e finalmente o bebê recusou o seio por estar acostumado com a mamadeira.

\section{Discussão}

Existem vários métodos possíveis de promover o aleitamento materno na comunidade. Já foi mostrado em São Paulo, que uma campanha bem organizada através da mídia pode ter efeito mensurável e positivo na amamentação ${ }^{16}$. Da mesma maneira uma política de saúde encorajando o aleitamento materno na comunidade e nos hospitais, com o auxílio dos profissionais de saúde, tem mostrado uma influência positiva nos padrões de aleitamento materno ${ }^{18,23}$.

Uma outra maneira efetiva de promover o aleitamento matemo é através das visitas domiciliares pós-parto. Neste momento as mães encontram dificuldades para amamentar e não podem contar com a ajuda dos profissionais do hospital, o que leva muitas vezes ao desmame precoce devido a falta de informação e orientação adequada. Certamente as visitas domiciliares pós-parto não representam uma idéia nova. Em 1937, o Parlamento da Dinamarca aprovou legislação que regulamentava a prática de visitas domiciliares pós-parto por profissionais de saúde com o objetivo de promover o aleitamento materno? ${ }^{7}$. Entretanto, muito poucos estudos usando metodologia adequada tem confirmado a efetividade desse método. Um ensaio controlado, realizado no País de Gales, mostrou que visitadoras domiciliares podem aumentar a prevalência de aleitamento materno. Nesse estudo, $84 \%$ das mães no grupo de intervenção estavam amamentando aos 4 meses após o parto contra $72 \%$ no grupo controle ${ }^{15}$. Na África do Sul, agentes comunitários foram utilizados com sucesso na promoção do aleitamento materno através de visitas domiciliares ${ }^{17}$.

O presente estudo mostra que visitas domiciliares pós-parto podem ter efeito positivo mensurável nos padrões de aleitamento materno nos primeiros meses de vida. É possível que se a intervenção fosse estendida por um período mais longo, com visitas no segundo e terceiro meses de vida, os efeitos do aleitamento poderiam ser reforçados. Este tipo de intervenção pode ser de bastante importância em áreas urbanas de muitos países em desenvolvimento, onde a alta hospitalar ocorre muito precocemente, e onde a educação a respeito de aleitamento é pouco realizada nos hospitais. No sul do Brasil, por exemplo, $25 \%$ das mães e bebês têm alta hospitalar nas primeiras 24 horas depois do nascimento, e $60 \%$ estão em casa após 48 horas depois do parto 3 .
Uma importante vantagem adicional é o baixo custo deste tipo de intervenção. Em nosso estudo, como os visitadores já trabalhavam como funcionários do sistema de saúde do município, apenas foram remanejados para esta função. Além disso, os custos diretos da intervenção foram mínimos.

Entretanto, mesmo que os visitadores tenham que ser contratados e treinados o custo-benefício da intervenção é positivo, principalmente se as visitas forem direcionadas para o grupo de crianças que apresentam maior risco para desmame precoce, e que no sul do Brasil são aquelas crianças pertencentes a famílias de baixa renda ${ }^{4}$.

O método utilizado para descobrir as principais causas de desmame foi de grande valia para tornar mais claro quais os elementos envolvidos na "Síndrome do leite insuficiente"14. Usando sistema de questões abertas e fechadas e categorizando as causas do desmame de maneira análoga ao atestado de óbito - subjacente, intermediária e imediata - verifica-se que o fato de 0 bebê chorar excessivamente é freqüentemente a causa básica do desmame. As mães relacionam, com freqüência, o choro à fome e então concluem que seu leite não é suficiente para satisfazer seu filho, tanto em volume quanto em qualidade. Esta cadeia de eventos está provavelmente relacionada à insegurança materna e à pressão dos familiares (principalmente as avós) e amigos. Da mesma maneira uma interpretação errônea das curvas de crescimento pode contribuir para esses eventos negativos. Como as crianças amamentadas podem ter um ritmo mais lento de ganho de peso após os três primeiros meses de vida, pode inferir-se equivocildamente que essas crianças estão crescendo de maneira indadequada, quando comparadas a crianças alimentadas com leite artificial ${ }^{11,22}$.

Esse fato é uma importante barreira na promoção do aleitamento materno, e é necessário que os profissionais de saúde estejam atentos para oferecer aconselhamento adequado às mães e suas famílias.

Fica evidenciado que os pais são bastante desinformados a respeito dos padrões normais de desenvolvimento infantil. Deveria sci mais divulgado que nos primeiros 3 meses de vida, o choro e irritação são comportamentos bastante comuns. Há uma tendência de aumentar o choro até as 6 semanas de vida e decrescer após este período ${ }^{2,8}$. A dificuldade de se adaptar ao choro excessivo leva os pais a tomarem a decisão mais fácil: substituir o leite materno por leite artificial. Mesmo com a aparente popularidade desta medida, Barr e col. ${ }^{12}$ mostraram que a diferença nos padrões de choro é na realidade uma redistribuição e não uma redução. Os bebês que trocaram o leite materno 
para leite artificial têm tendência a chorar menos durante a noite, mas não menos durante a manhã. Este fato reflete mudanças em padrões de sono o que aumenta o número de horas de sono noturno para os bebês que desmamam ${ }^{12}$. Estes achados confirmam que a mudança de alimentação não é a solução para o choro excessivo. O método mais efetivo para isso é a promoção do contato físico dos bebês com suas mães ${ }^{6}$. Barr e Elias ${ }^{1}$ mostraram que períodos curtos entre as mamadas e resposta materna rápida às necessidades do bebê estão associadas com menos choro nos primeiros meses de vida. Ao lado da informação clínica adequada, as campanhas da mídia devem também fornecer informações sobre os padrões normais de comportamento do lactente e sobre a interação mãe-bebê, deixando bem evidente que o choro do lactente nas primeiras semanas de vida é parte de seu desenvolvimento normal, e não quer dizer necessariamente que está com fome e o leite materno é insuficiente.

\section{Agradecimento}

Dr. Jay McAuliffe (Projeto Hope, Ceará, Brasil) pelos seus valiosos comentários na versão preliminar deste artigo.

BARROS, F.C. et al. [A randomized intervention trial to increase breast-feeding prevalence in southern Brazill. Rev. Saúde Pública, 28: 277-83, 1994. A randomized intervention trial to promote breast-feeding was carried out in southern Brazil. A group of 450 mothers and babies was visited at home 5, 10 and 20 days after birth and compared to a non-visited control group of the same size. Ninety-two per cent of the families visited received the three home visits planned. The evaluation of breast-feeding patterns and reasons for weaning took place 6 months after birth for both groups. Ninety-four per cent of the group visited and $92 \%$ of the non-visited controls group were traced on the occasion of the assessment. The intervention increased the duration of breastfeeding (median duration of 120 days in the group visited and 105 days in the controls; $p=0.03$ ) and delayed the introduction of milk bottles (median age of introduction of 90 days in the group visited and 60 days in the controls; $p=0.01$ ). Causes of weaning were classified as underlying, intermediate and immediate. The most common underlying cause of weaning was "the baby cried too much", which suggests that mothers should be taught about normal patterns of infant behaviour in the first weeks of life, particularly the need for crying, and the fact that this not necessarily reflects hunger.

Keywords: Breast feeding. Health promotion, methods. Intervention studies.

\section{Referências Bibliográficas}

1. BARR, G.R. \& ELIAS, F.M. Nursing interval and maternal responsivity: effect on early infant crying. Pediatrics, 81: 529-36, 1988.

2. BARR, G.R.; KRAMER, S.M.; PLESS, B.L.; BOISJOLY, C.; LEDUC, D. Feeding and temperament as determinants of early infant crying fussing behavior. Pediatrics, 84: 514-21, 1989.

3. BARROS, F.C. Perinatal health in Southem Brazil. A study of perinatal mortality, low birth weigth and the utilization of health care. London,1985 [PhD Thesis University of London]

4. BARROS, F.C.; VICTORA, C.G.; VAUGHAN, J.P. Breastfeeding and socioeconomic status in Southern Brazil. Acta Paediatr. Scand., 75:558-62,1986.

5. BARROS, F.C.; VICTORA, C.G.; VAUGHAN, J.P.; TEIXEIRA, A.M.B.; ASHWORTH, A. Infant mortality in Southern Brazil: a population based study of causes of death. Arch. Dis. Child., 62: 487-90, 1987.

6. BELL, S.M. \& AINSWORTH, M.D.S. Infant crying and maternal responsiveness. Child Dev., 43: 1171-90, 1972.

7. BIERING-SORENSEN, F.; HILDEN, J.; BIERINGSORENSEN, K. Breast-feeding in Copenhagen, 19381977. Dan. Med. Bull., 27: 42-8, 1980.

8. BRAZELTON, T.B. Crying in infancy. Pediatrics, 29: 57988, 1962.

9. BROWN, K.H.; BLACK, R.E.; ROMANA, G.L.; KANASHIRO, H.C. Infant-feeding practices and their relationship with diarrheal and other diseases in Huascar (Lima), Peru. Pediatrics, 83: 31-40, 1989.

10. CUNNINGHAN, A.S.; JELLIFFE, D.B.; JELLIFFE, E.F.P. Breast-feeding and health in the 1980's: a global epidemiologic review. J.Pediatr., 118: 659-66, 1991.

11. DEWEY, K.G.; HEINIG, J.; NOMMSEN, A.L.; PEERSON, M.J.; LONNERDAL, B. Growth of breast-fed and formula-fed infants from 0 to 18 months: the Darling study. Pediatrics, 89: 1035-41, 1992.

12. ELIAS, F.M.; NICOLSON, A.N.; BORA, C.; JOHNSTON, $J$. Sleep wake patterns of breast-fed infants in the first 2 years of life. Pediatrics, 77: 322-9, 1986.

13. EPILOG PLUS. Epicenter Software. Pasadena, Ca., 1985.

14. GUSSLER. J.D. \& BRIESEMEISTER, L.H. The insufficient milk syndrome: a biocultural explanation. Med. Anthropol., 4: 1-24, 1980.

15. JONES, D.A \& WEST, R.R. Effect of lactation nurse on the success of breast-feeding: a randomized controlled trial. J. Epidemiol. Community Health, 40: 45 9, 1986.

16. REA, M.F. The Brazilian National Breastfeeding Programme: a success history. Int. J. Gynecol. Obstet., 31 (Suppl. 1): 79.82, 1990.

17. ROSS, S.M.; LOENING, W.E.; MBELE, B.E. Breastfeeding support. S. Afr. Med. J., 72: 357-8, 1987.

18. SLOPER, K.S.; ELSDEN, E.; BAUM, J.D. Increasing breast feeding in a community. Arch. Dis. Child., 52: 700-2, 1977.

19. VICTORA, C.G.; SMITH, P.G.; VAUGHAN, J.P.; NOBRE, L.C.; LOMBARDI, C.; TEIXEIRA, A.M.B.; FUCHS, S.M.C.; MOREIRA, L.B.; GIGANTE, L.P.; BARROS, F.C. Evidence for a strong protective effect of breast-feeding against infant deaths from infectious diseases in Brazil. Lancet, 2: 319-22, 1987.

20. VICTORA, C.G.; BARROS, F.C.; VAUGHAN, J.P. TEIXEIRA, A.M.B. Birthweigth and infant mortality. A longitudinal study of 5914 Brazilian children. Int. J. Epidemiol., 16: 239-45, 1987. 
21. VICTORA, C.G.; SMITH, P.G.; VAUGHAN, J.P.; NOBRE, L.C.; LOMBARDI, C.; TEIXEIRA, A.M.B.; FUCHS, S.M.C.; MOREIRA, L.B.; GIGANTE, L.P.; BARROS, F.C. Infant feeding and deaths due to diarrhoea: a case-control study. Am. J. Epidemiol., 129: 1032-41, 1989.

22. WHITEHEAD, R.G. \& PAUL, A.A. Growth charts and the assessment of infant feeding practices in the Western world and in developing countries. Early Hum. Dev., 9: 187-207, 1984.

23. WINICOFF, B. \& BAER, E.C. The obstetrician's opportunity: translating "breast in best" from theory to practice. Am. J. Obstet. Gynecol., 138: 105-17, 1980.

Recebido para publicação em 13.1.1994 Aprovado para publicaçâo em 20.5.1994 